\title{
Confocal Laser Endomicroscopic Findings of Refractory Erosive Reflux Disease versus Non-Erosive Reflux Disease with Anti-Reflux Mucosectomy: An in vivo and ex vivo Study
}

\author{
Eunju Jeong ${ }^{1 *}$, In Kyung Yoo ${ }^{1 *}$, Abdullah Özgür Yeniova ${ }^{2}$, Dong Keon Yon ${ }^{3}$ and Joo Young Cho ${ }^{1}$ \\ ${ }^{1}$ Department of Gastroenterology, CHA Bundang Medical Center, CHA University College of Medicine, Seongnam, Korea, ${ }^{2}$ Division of \\ Gastroenterology, Department of Internal Medicine, Tokat Gaziosmanpaşa University, Faculty of Medicine, Tokat, Turkey, ${ }^{3}$ Department \\ of Pediatrics, CHA Bundang Medical Center, CHA University College of Medicine, Seongnam, Korea
}

Background/Aims: To date, there is no standard tool to diagnose gastroesophageal reflux disease (GERD). Typically, GERD is a non-erosive reflux disease (NERD) that does not present endoscopic abnormalities. Confocal laser endomicroscopy (CLE) has been shown to be an effective tool to identify and diagnose GERD. We aimed to investigate the cellular and vascular changes in vivo and ex vivo through CLE in patients with GERD.

Methods: Patients with refractory GERD who underwent mucosectomy were recruited. The distal esophagus was observed in vivo using CLE. Mucosectomy tissue was stained with acriflavine and CLE image was obtained ex vivo. We compared cellular and vascular changes in CLE between erosive reflux disease (ERD), NERD, and a control group.

Results: Eleven patients who underwent anti-reflux mucosectomy and five control patients were enrolled in the study. Patients with ERD and NERD presented greater dilated intercellular space than patients in the control group on CLE image. The diameter, number, and cross-sectional area of the intra-papillary capillary loops (IPCLs) were significantly larger in the ERD group than in the NERD group. The irregular shape of the IPCLs were observed in both patients with ERD and NERD.

Conclusions: The irregular shape of the IPCLs were significantly correlated with a positive diagnosis of GERD. CLE may diagnose NERD with high sensitivity and accuracy. Clin Endosc 2021;54:55-63

Key Words: Anti-reflux mucosectomy; Confocal laser endomicroscopy; Erosive reflux disease; Non-erosive reflux disease

\section{INTRODUCTION}

Recently, there has been an increased incidence of gastroesophageal reflux disease (GERD), which is associated with reduced quality of life due to repeated exacerbations resulting in adverse events. ${ }^{1}$ GERD is usually diagnosed through reflux symptoms, endoscopy, and 24-hour $\mathrm{pH}$ monitoring, how-

Received: February 6, 2020 Revised: March 30, 2020

Accepted: April 1, 2020

Correspondence: Joo Young Cho

Digestive Disease Center, CHA Bundang Medical Center, CHA University College of Medicine, 59 Yatap-ro, Bundang-gu, Seongnam 13496, Korea

Tel: +82-31-780-5641, Fax: +82-32-780-5005, E-mail: cjy6695@dreamwiz.com

ORCID: https://orcid.org/0000-0001-9085-0313

*These authors contributed equally to this study.

(cc) This is an Open Access article distributed under the terms of the Creative Commons Attribution Non-Commercial License (http://creativecommons.org/ licenses/by-nc/3.0) which permits unrestricted non-commercial use, distribution, and reproduction in any medium, provided the original work is properly cited. ever, no standard diagnostic tools have yet been established. ${ }^{2}$ Based on the Los Angeles classification system (LA system), GERD can be classified as an erosive reflux disease (ERD; reflux esophagitis) or a non-erosive reflux disease (NERD), depending on the severity of the mucosal erosion or break. ${ }^{3}$ Despite the presence of reflux-associated symptoms, cases in which a definitive mucosal break is not observed on endoscopy are considered NERD. ${ }^{4}$ A modified LA system, which includes minimal change (grade $\mathrm{M}$; erythema and/or whitish turbidity), is used in Japan; however, inter observer agreement among endoscopists is low, the relationship between symptoms and pathology is unclear, and it is not internationally accepted. ${ }^{5}$ Technological advances in endoscopic procedures have enabled the observation of subtle mucosal changes to the gastroesophageal junction (GEJ) using magnification endoscopy, chromoscopy, narrow-band imaging (NBI), and flexible spectral imaging color enhancement. In addition, many studies have examined the correlation between endoscopy 
and histopathological findings. ${ }^{6,7}$ The introduction of confocal laser endomicroscopy (CLE) has enabled the observation of in vivo cellular and vascular changes at the GEJ during endoscopy in real-time. ${ }^{8,9}$ When acid exposure occurs repeatedly and reflux esophagitis develops, histopathological changes in the squamous cell epithelium of the distal esophagus occur (such as elongated papillae, basal cell proliferation, dilated intercellular space [DIS], increased number of intra-papillary capillary loops [IPCLs], and IPCL diameter). ${ }^{10-12}$ Some studies compared these histological differences among ERD, NERD, and functional heartburn using $\mathrm{CLE},{ }^{2}$ while others investigated histological changes with and without proton pump inhibitors (PPIs). ${ }^{13,14}$

In refractory GERD, patients fail to respond to conventional acid suppressing PPI therapy. ${ }^{15}$ Anti-reflux mucosectomy (ARMS) was recently introduced as a minimally invasive treatment for refractory GERD. ${ }^{16}$ This study aimed to investigate the in vivo and ex vivo CLE findings of subtle cellular and vascular changes of the GEJ in patients diagnosed with GERD and compare the CLE findings between patients with NERD, $\mathrm{ERD}$, and a control group.

\section{MATERIALS AND METHODS}

\section{Patients}

From March to November 2018, 11 patients with refractory GERD were recruited from the Endoscopy Unit of CHA Bundang Medical Center, Seongnam, Republic of Korea. Refractory GERD was defined as chronic reflux symptoms (heartburn and regurgitation as typical symptom; chest discomfort, epigastric pain, halitosis, and hoarseness as atypical symptoms) that were not reduced or alleviated to less than $50 \%$ despite double-dose PPI therapy for at least 12 weeks. ${ }^{17}$ All patients provided informed consent for the procedure. Screening tests, such as the GERD-Q, conventional white light endoscopy (WLE), 24-hour $\mathrm{pH}$ monitoring, high-resolution manometry (HRM), and endolumenal functional lumen imaging probe (EndoFLIP; Medtronic, Minneapolis, MN, USA), were performed before ARMS.

All patients were first examined by WLE. Patients were classified according to their endoscopic findings. Patients diagnosed with refractory GERD with esophageal mucosal injury (mucosal erosion and break) on endoscopy were classified as ERD. Meanwhile, patients diagnosed with refractory GERD with no evidence of esophageal mucosal injury on endoscopy were classified as NERD. Patients with normal esophageal $\mathrm{pH}$ test and with evidence of a close correlation between patients' heartburn and reflux events (reflux hypersensitivity) were also recruited to the study, and these patients were classified as
ERD or NERD based on their endoscopic findings. Patients with functional heartburn were excluded from the study. We recruited controls who did not have reflux-associated symptoms or a mucosal break on conventional WLE, but who underwent endoscopy for other indications. All patients stopped PPI or $\mathrm{H}_{2}$ receptor antagonist medication for at least 4 weeks before screening. Patients aged 33-75 years who underwent ARMS for refractory GERD were included in this study. The exclusion criteria were as follows: (1) refusal to provide study consent, (2) severe or uncontrolled medical condition (heart or lung disease, acute severe infection, etc.), (3) history of esophageal surgery or disease (cancer, varices, stricture, lipoma, cyst, submucosal tumor, etc.), (4) use of systemic steroids or nonsteroidal anti-inflammatory drugs within 2 weeks prior to the procedure, (5) severe coagulopathy, (6) impaired kidney or liver function, (7) history of allergy (drug- or food-induced cutaneous eruption, allergic rhinitis, allergic asthma, etc.) and drug-induced adverse reactions after fluorescein injection and (8) functional heartburn. All procedures were conducted in accordance with the ethical standards of the Hospital Ethics Committee and Institutional Review Board of CHA University (approval number: CHAMC 2019-02-034-003).

\section{Endoscopy and confocal laser endomicroscopy procedures}

All procedures were performed using the probe-based CLE (pCLE; Cellvizio series 100; Mauna Kea Technologies, Paris, France) before and after ARMS. Ex vivo CLE was performed using specimens obtained from ARMS in patients with refractory GERD.

First, all patients underwent WLE before the pCLE. Subsequently, $0.5 \mathrm{~mL}$ of $10 \%$ fluorescein was subcutaneously injected, and the patients were observed for 30-60 min for allergic reactions. Next, intravenous sedation with propofol and midazolam was administered and cardiopulmonary monitoring was performed during the procedure. Before the ARMS, 2.5-5 $\mathrm{mL}$ of $10 \%$ fluorescein was intravenously injected, and a real-time image was observed and measured in every quadrant of the distal $2 \mathrm{~cm}$ of the esophageal mucosa above the Z-line through the pCLE in vivo (Supplementary Video 1). The tissue obtained during the ARMS was immediately stained with acriflavine (acriflavine hydrochloride) or acrinol solution (ethacridine lactate) for 5 minutes and then observed ex vivo by pCLE (fluorescein does not stain the nuclei, therefore acriflavine was used to observe the nuclei of the esophageal epithelium. Acriflavine is not used directly in the human body due to adverse events; Supplementary Video 2). The pCLE procedures were performed by two experienced endoscopists (IKY and JYC) who had performed $>100$ pCLE procedures prior to the present study. 


\section{Study measurement}

This non-randomized pilot study aimed to compare vascular and cellular changes in vivo and ex vivo between refractory ERD and NERD using pCLE. Four ERD patients, 7 NERD patients, and 5 controls were recruited.

Study measurements were as follows:

1. DISs in the epithelium;

2. Number, diameter, and cross-sectional areas (CSAs) of the IPCLs, and

3. Presence or absence of irregularly shaped IPCLs.

We saved the pCLE movie and then captured all the images and measured them manually using the Image J program. The diameters and CSAs of the IPCLs and DISs in all epithelial samples seen in the captured image were measured, and the median value was obtained after exclusion of the smallest and largest values. The median value was measured by manual counting of the number of IPCLs seen in all captured images. The irregular shape of IPCLs was identified by the presence or absence of elongation and tortuosity. Subsequently, we analyzed and compared the subtle cellular and vascular changes in the ERD, NERD, and control groups.

\section{Anti-reflux mucosectomy procedure}

ARMS was performed using the endoscopic mucosal resection-cap (EMRC) technique. Based on this method, approximately $60 \%-70 \%$ of the esophagogastric junction (EGJ) circumference was resected. The EGJ were marked using an argon plasma coagulation unit (30 W; APC2 VIO 300D; Erbe Medical Systems, Tübingen, Germany) at the 10 and 6 oclock directions prior to performing the procedure on the lesser curve of the stomach. After administering a submucosal injection using saline with indigo carmine dye, EMRC was performed repeatedly until the marked mucosal area was completely resected (Fig. 1). The result is mucosal flap valve remodeling as an effective anti-reflux mechanism at the anatomical level. Unlike other anti-reflux treatments, the EMRC

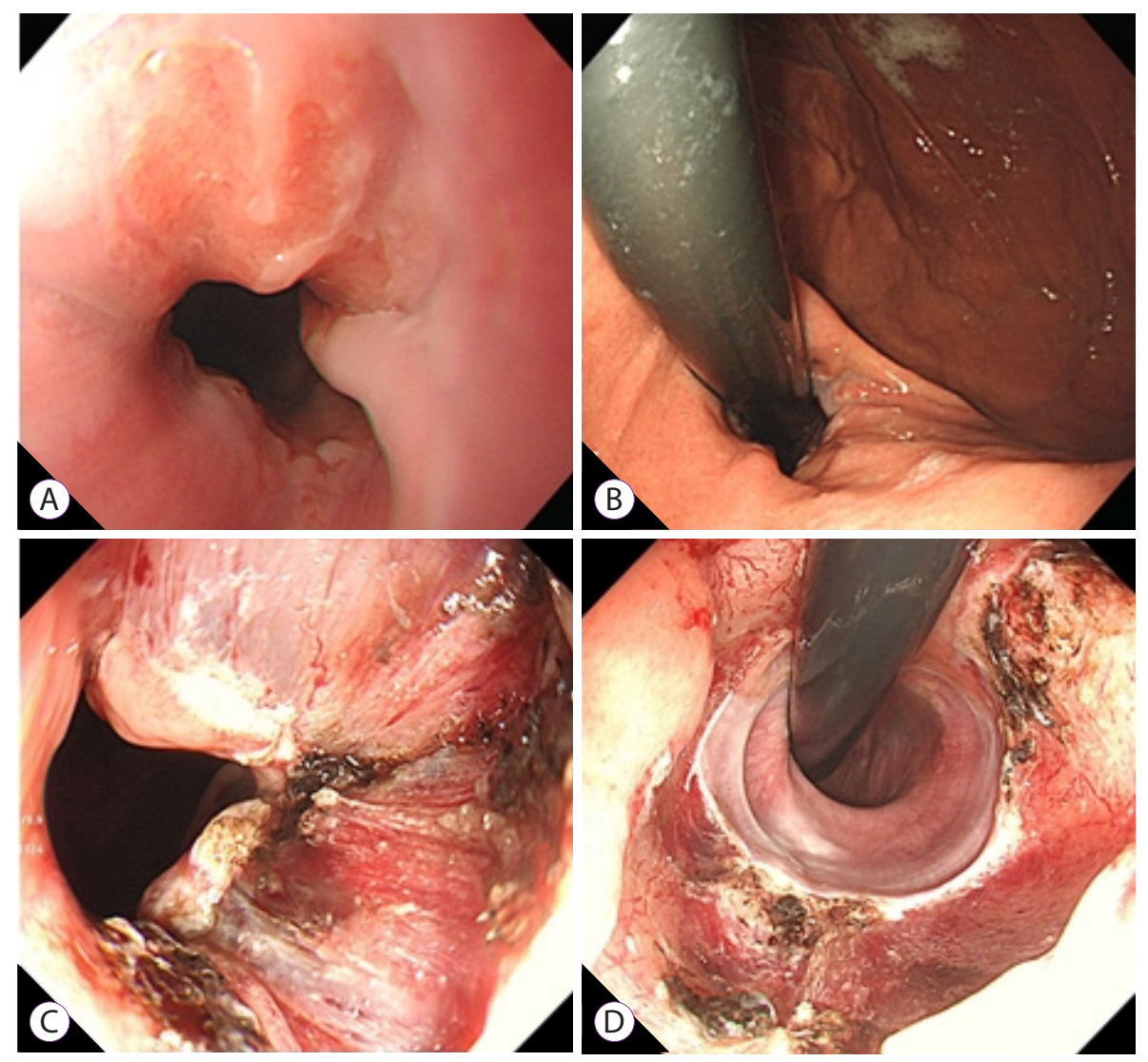

Fig. 1. Anti-reflux mucosectomy. (A) Forward view of the esophagogastric junction (EGJ) before the procedure; (B) Retroflexed view of the EGJ before the procedure; (C) Forward view of the EGJ immediately after the procedure (using endoscopic mucosal resection-cap); (D) Retroflexed view of the EGJ immediately after the procedure. 
technique allows physicians to obtain the mucosectomy tissue for evaluation. All ARMS procedures were performed by experienced endoscopists (JYC and IKY) who had previously performed more than 400 endoscopic resections annually.

\section{Statistical analysis}

Values were expressed as median $\pm \mathrm{SD}$. The statistical analysis was performed using SPSS Statistics 25 (SPSS, IBM Co., Armonk, NY, USA). The patients' baseline characteristics and in vivo CLE findings with NERD and ERD were compared to those without NERD and ERD (control) using the one-way ANOVA, student $t$-test, or Fisher's exact test. EndoFLIP, HRM, and 24-hour $\mathrm{pH}$ monitoring were analyzed by student $t$-test. The ex vivo CLE findings were analyzed using the ANCOVA, with the baseline value of age and sex as the covariate. The values were considered statistically significant when $p<0.05$.

\section{RESULTS}

\section{Patients' baseline characteristics}

After the exclusion of 6 patients with poor pCLE images who had taken a PPI within 48 hours prior to the procedure, 22 patients were included in this study. We enrolled 4 patients who underwent ARMS in the ERD group, 7 patients who underwent ARMS in the NERD group, and 5 patients in the control group. The patients' demographic and clinical characteristics are shown in Table $1(p>0.05)$. Although typical

Table 1. Patients' Baseline Characteristics

\begin{tabular}{|c|c|c|c|c|}
\hline Characteristics & $\begin{array}{c}\text { ERD group } \\
(n=4)\end{array}$ & $\begin{array}{c}\text { NERD group } \\
(n=7)\end{array}$ & $\begin{array}{c}\text { Control group } \\
(n=5)\end{array}$ & $p$-value \\
\hline Sex (Male:Female) & $3: 1$ & $6: 1$ & $2: 3$ & $0.327^{\mathrm{a})}$ \\
\hline Age, yr & $52.50 \pm 3.69$ & $48.43 \pm 11.70$ & $63.20 \pm 12.87$ & $0.268^{\mathrm{b})}$ \\
\hline Weight, kg & $72.72 \pm 18.98$ & $65.74 \pm 13.43$ & $61.85 \pm 7.75$ & $0.505^{\mathrm{b})}$ \\
\hline BMI, $\mathrm{kg} / \mathrm{m}^{2}$ & $27.26 \pm 8.21$ & $21.90 \pm 3.40$ & $24.28 \pm 2.19$ & $0.230^{\mathrm{b})}$ \\
\hline Smoking & 0 & 3 & 0 & $0.141^{\text {a) }}$ \\
\hline Alcohol drinking & 1 & 3 & 0 & $0.999^{\mathrm{a})}$ \\
\hline Main symptom & & & & $0.005^{\mathrm{a})}$ \\
\hline Heartburn & 2 & 1 & 0 & \\
\hline Regurgitation & 1 & 2 & 0 & \\
\hline Epigastric pain & 1 & 2 & 0 & \\
\hline Halitus & 0 & 1 & 0 & \\
\hline Hoarseness & 0 & 0 & 0 & \\
\hline Chest discomfort & 0 & 1 & 0 & \\
\hline Disease duration, yr & $14.75 \pm 12.52$ & $6.27 \pm 10.52$ & - & $0.259^{c)}$ \\
\hline LA classification & & & & $<0.001^{\text {a) }}$ \\
\hline Normal & 0 & 7 & 5 & \\
\hline A to $\mathrm{D}$ & 4 & 0 & 0 & \\
\hline Hiatal hernia & 4 & 3 & 0 & \\
\hline GERD-Q score & $14.00 \pm 2.45$ & $10.57 \pm 3.35$ & - & $0.110^{\mathrm{c})}$ \\
\hline Range of mucosectomy, \% & $65.00 \pm 5.77$ & $70.00 \pm 5.77$ & - & $0.200^{c)}$ \\
\hline \multicolumn{5}{|l|}{ Final histopathology } \\
\hline Chronic gastritis without IM & 3 & 5 & - & \\
\hline Chronic gastritis with IM & 1 & 2 & - & \\
\hline
\end{tabular}

BMI, body mass index; ERD, erosive reflux disease; GERD, gastroesophageal reflux disease; IM, intestinal metaplasia; NERD, non-erosive reflux disease.

${ }^{a)} p$-value calculated by using the Fisher's exact test.

${ }^{b)} p$-value calculated by using the one-way ANOVA.

${ }^{c)} p$-value calculated by using the student $t$-test. 
symptoms such as heartburn and regurgitation were predominant in the ERD and NERD groups, various atypical symptoms (substernal chest pain, halitus, epigastric pain, etc.) were also present. The mean disease duration was slightly longer in the ERD group than in the NERD group, and the GERD-Q questionnaire score was higher; however, the difference was not statistically significant. Based on the LA classification criteria in WLE, all ERD groups were grade C. Barrett esophagus was diagnosed in 2 patients (50\%) in the ERD group. The WLE findings in all groups are shown in Fig. 2. Table 2 shows the baseline results of EndoFLIP, HRM, and 24-hour pH monitoring before ARMS in the ERD and NERD groups. The distensibility index of the EndoFLIP was higher in the ERD group than in the NERD group, but the difference was not statistically significant. The lower esophageal sphincter pressure and the integrated relaxation pressure in the HRM did not differ between the two groups. The 24-hour $\mathrm{pH}$ monitoring showed that the DeMeester score and total acid exposure time were significantly higher in the ERD group than in the NERD group ( $p=0.002$ and $p=0.005$, respectively).

\section{In vivo and ex vivo comparisons of cellular and vascular changes on confocal laser endomicroscopy}

Table 3 compares the in vivo cellular and vascular changes among the ERD, NERD, and control groups. A real-time image was taken of every quadrant of the distal $2 \mathrm{~cm}$ of the esophageal mucosa above the Z-line through pCLE in vivo for analysis. The DISs did not differ significantly between the ERD and NERD groups ( $5.49 \pm 0.68$ vs. $5.03 \pm 1.55$, respectively, $p=0.794$ ), however, there was a significant difference be-

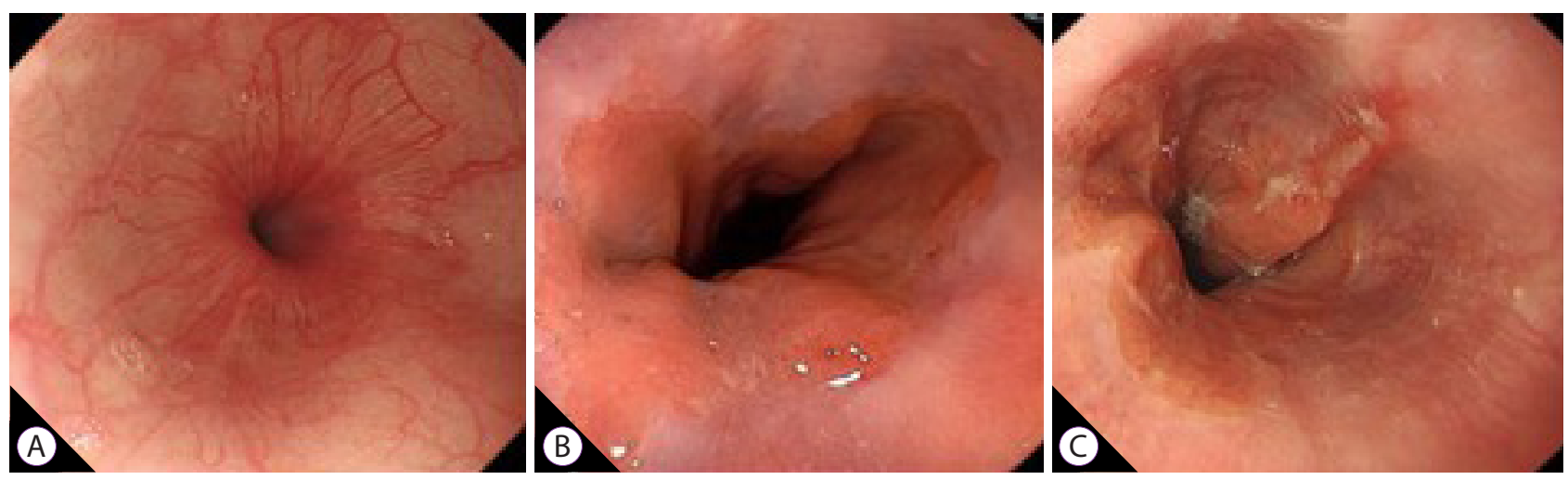

Fig. 2. White light endoscopy findings: (A) normal (control group); (B) minimal change esophagitis (non-erosive reflux disease group); (C) erosive esophagitis (erosive reflux disease group).

Table 2. EndoFLIP, High-Resolution Manometry, and 24-Hour pH Monitoring in the Erosive Reflux Disease versus Non-Erosive Reflux Disease Groups

\begin{tabular}{|c|c|c|c|}
\hline & $\begin{array}{c}\text { ERD group } \\
(n=4)\end{array}$ & $\begin{array}{l}\text { NERD group } \\
(n=7)\end{array}$ & $p$-value \\
\hline \multicolumn{4}{|l|}{ EndoFLIP } \\
\hline $\mathrm{DI}_{40}, \mathrm{~mm}^{2} / \mathrm{mm} \mathrm{Hg}$ & $15.75 \pm 5.23$ & $10.84 \pm 7.07$ & 0.260 \\
\hline $\mathrm{CSA}, \mathrm{mm}^{2}$ & $213.65 \pm 41.64$ & $190.81 \pm 102.19$ & 0.744 \\
\hline \multicolumn{4}{|l|}{ High-resolution manometry } \\
\hline LES pressure, $\mathrm{mm} \mathrm{Hg}$ & $15.25 \pm 7.08$ & $13.33 \pm 3.21$ & 0.836 \\
\hline IRP, mm Hg & $8.50 \pm 2.51$ & $6.28 \pm 2.69$ & 0.768 \\
\hline \multicolumn{4}{|l|}{ 24-hour $\mathrm{pH}$ monitoring } \\
\hline DeMeester score $($ normal <14.7) & $13.85 \pm 11.00$ & $4.92 \pm 4.74$ & 0.002 \\
\hline Total acid exposure time, $\%$ (normal $<4.2$ ) & $3.85 \pm 3.12$ & $1.32 \pm 1.43$ & 0.005 \\
\hline Total bolus exposure time, \% (normal <1.4) & $3.77 \pm 1.69$ & $1.51 \pm 1.21$ & 0.221 \\
\hline
\end{tabular}

$p$-value calculated by using the student $t$-test.

CSA, cross-sectional area; DI, distensibility index; ERD, erosive reflux disease; IRP, integrated relaxation pressure; LES, lower esophageal sphincter; NERD, non-erosive reflux disease. 

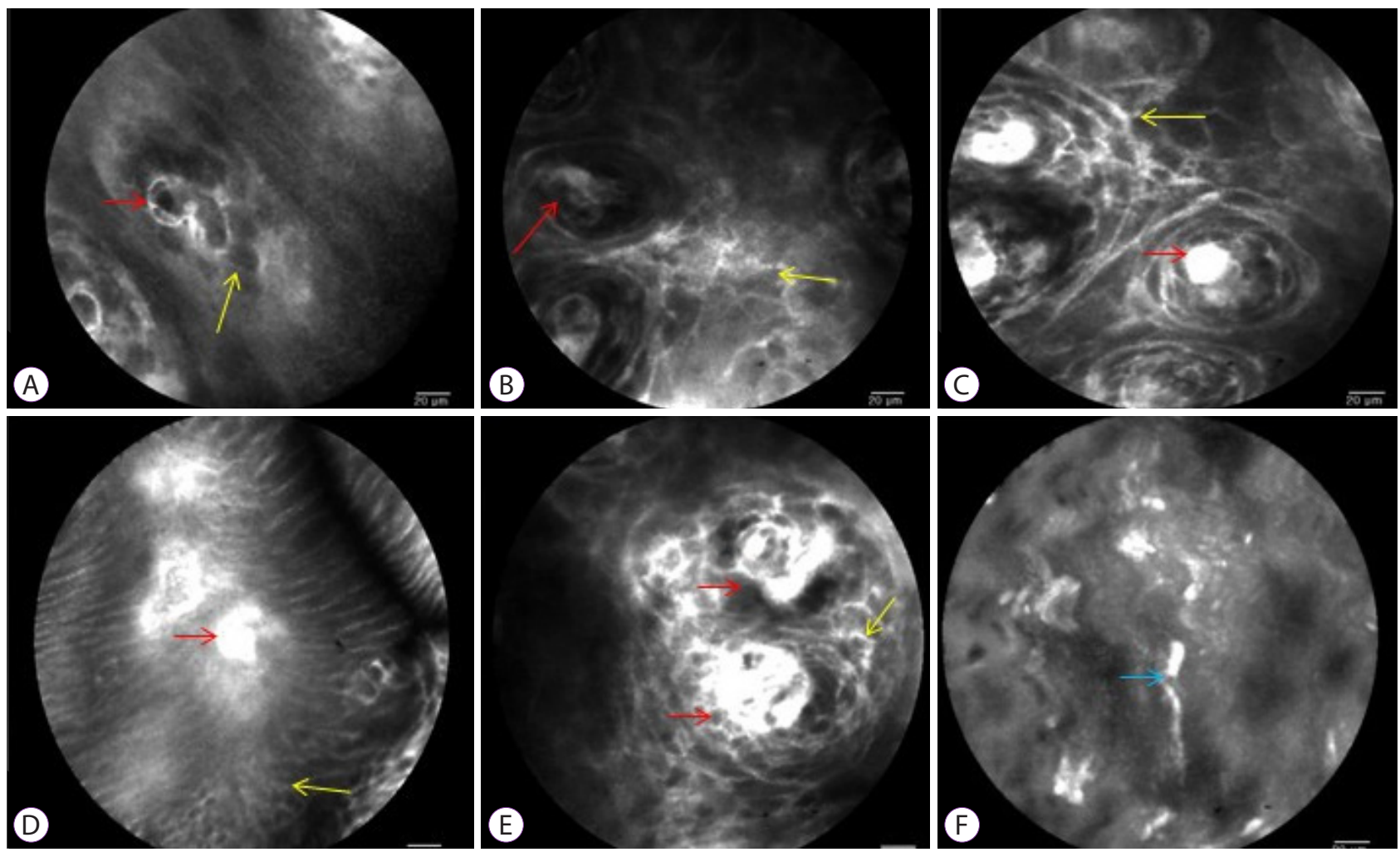

Fig. 3. Confocal laser endomicroscopy findings. (A) normal (control group); ( $B, D$ ) non-erosive reflux disease (NERD) group; (C, E) erosive reflux disease (ERD) group. The red arrow indicates intra-papillary capillary loops (IPCLs) and the diameter and number of IPCLs were higher from NERD to ERD group, compared with the control group. The yellow arrow indicates that the dilated intercellular spaces of epithelium was increased, but the differences between the NERD and ERD groups are not significant (A to E). The light blue arrows indicate the elongation of the IPCLs, which was observed in the ERD and NERD groups, but not in the control group (F).

Table 3. Cellular and Vascular Changes on Confocal Laser Endomicroscopy (in vivo)

\begin{tabular}{lcccc}
\hline & $\begin{array}{c}\text { ERD group } \\
(\boldsymbol{n}=\mathbf{4})\end{array}$ & $\begin{array}{c}\text { NERD group } \\
(\boldsymbol{n}=7)\end{array}$ & Control group & $\boldsymbol{p}^{\text {-value }}$ \\
\hline Dilated intercellular space, $\mu \mathrm{m}$ & $5.49 \pm 0.68$ & $5.03 \pm 1.55$ & $2.50 \pm 0.33$ & 0.002 \\
IPCLs & & & & \\
$\quad$ Number, $n$ & $7.15 \pm 1.48$ & $4.93 \pm 1.44$ & $2.71 \pm 0.87$ & $0.001^{\mathrm{a})}$ \\
Diameter, $\mu \mathrm{m}$ & $21.98 \pm 7.01$ & $10.96 \pm 1.50$ & $6.36 \pm 1.03$ & $<0.001^{\mathrm{a})}$ \\
CSA, $\mu \mathrm{m}^{2}$ & $260.29 \pm 146.43$ & $121.72 \pm 45.15$ & $50.40 \pm 19.92$ & $0.005^{\mathrm{a})}$ \\
Presence of irregularly shaped IPCLs, $n$ & 4 & 7 & 0 & 0.001 \\
\hline
\end{tabular}

CSA, cross-sectional area; ERD, erosive reflux disease; IPCLs, intra-papillary capillary loops; NERD, non-erosive reflux disease. a) $p$-value calculated by using the one-way ANOVA.

tween the ERD and control groups $(5.49 \pm 0.68$ vs. $2.50 \pm 0.33$, $p=0.004)$ and between the NERD and control groups $(5.03 \pm 1.55$ vs. $2.50 \pm 0.33, p=0.005)$. The diameter, number, and CSA of the IPCLs were significantly higher in the ERD group than in the NERD group. Irregularly shaped IPCLs were observed in the ERD and NERD groups, but not in the control group (Table 3, Fig. 3).
In the ERD and NERD groups, tissue samples obtained after ARMS were observed ex vivo for cellular and vascular changes via pCLE. Similarly, as with the in vivo finding, DISs did not differ significantly between the ERD and NERD groups $(9.01 \pm 3.09$ vs. $6.68 \pm 2.65, p=0.073)$. Additionally, the diameter of the IPCLs was significantly higher in the ERD group than in the NERD group. The number, and CSA of the IPCLs, 
Table 4. Cellular and Vascular Changes on Confocal Laser Endomicroscopy (ex vivo)

\begin{tabular}{lccc}
\hline & $\begin{array}{c}\text { ERD group } \\
(\boldsymbol{n}=\mathbf{4})\end{array}$ & $\begin{array}{c}\text { NERD group } \\
(\boldsymbol{n}=7)\end{array}$ & $\boldsymbol{p}$-value \\
\hline Dilated intercellular space, $\mu \mathrm{m}$ & $9.01 \pm 3.09$ & $6.68 \pm 2.65$ & 0.218 \\
IPCLs & & & 0.001 \\
$\quad$ Number, $n$ & $11.45 \pm 2.42$ & $6.41 \pm 1.26$ & 0.102 \\
Diameter, $\mu \mathrm{m}^{2}$ & $29.81 \pm 9.74$ & $18.49 \pm 1.74$ & 0.010 \\
CSA, $\mu \mathrm{m}^{2}$ & $331.86 \pm 104.48$ & $186.23 \pm 47.27$ & N/A \\
Presence of irregularly shaped IPCLs, $n$ & 4 & 7 & \\
\hline
\end{tabular}

CSA, cross-sectional area; ERD, erosive reflux disease; IPCLs, intra-papillary capillary loops; N/A, not available; NERD, non-erosive reflux disease.

were significantly higher in the ERD group than in the NERD group. Irregularly shaped IPCLs were observed in the ERD and NERD groups (Table 4, Fig. 3). A CLE image is shown in Fig. 3. The red arrow indicates IPCLs, and the diameter and number of IPCLs were higher from the NERD to the ERD group, compared with the control group. The yellow arrow indicates that the DISs were widening, but the differences between the NERD and ERD groups was not significant. The light blue arrows indicate IPCL elongation, which was observed in the ERD and NERD groups, but not in the control group.

In summary, in vivo findings indicated that there was no difference between the ERD and NERD groups in the mean of the DIS, however, both groups had larger DISs than the control group. The number and CSA of the IPCLs in the ERD group were higher than those in NERD and control groups. The diameter of the IPCLs in the ERD group was significantly higher than that in the NERD and control groups. Both NERD and ERD groups had irregular shaped IPCLs, but no irregular shaped IPCL was observed in the control group.

Ex vivo findings indicated that there was no difference between the NERD and ERD groups in the mean of DISs. The number and CSA of the IPCL in the ERD group were significantly higher than those in the NERD group. The diameter of the IPCL in the ERD group was significantly larger than that in the NERD group. However, both groups had irregular-shaped IPCLs.

\section{DISCUSSION}

GERD is a chronic disease that is caused by the reflux of gastric contents. Anti-acid medications are used for symptom relief, however, to date, no definitive medical therapy exists for this disease. Furthermore anti-acid medications have been associated with various side effects. ${ }^{18}$
Treatments that aim to create an anti-reflux barrier were used for three decades. For example, laparoscopic fundoplication is highly effective but may result in complications including dysphagia, wound infection, and pneumonia. Mucosal resection was recently introduced as an endoscopic anti-reflux modality to treat refractory GERD. ${ }^{16}$ ARMS is an emerging technique with good subjective and objective outcomes for the treatment of GERD. ${ }^{19}$ However, outcome data of endoscopic mucosectomy techniques is very limited and long-term studies are needed.

NERD is responsible for the majority of GERD cases, and conventional WLE findings have low agreement between observers and there exists no clear diagnostic criteria. ${ }^{5}$ To date, many studies have used endoscopy and histopathology to elucidate NERD diagnoses. ${ }^{10-12}$ NERD causes refractory GERD due to its poor response to acid suppression therapy. ${ }^{15} \mathrm{NERD}$, ERD, reflux hypersensitivity, and functional heartburn present with similar symptoms (heartburn, regurgitation, chest pain, cough, hoarseness, etc.). Differential diagnosis of NERD, ERD, and other diseases is important to prevent patients from undergoing unnecessary anti-acid therapy. Some patients' symptoms may improve with anti-reflux barrier treatment (laparoscopic surgery or endoscopic procedures). Other diagnostic modalities that differentiate NERD, ERD, reflux hypersensitivity, and functional heartburn are HRM, 24-hour $\mathrm{pH}$ monitoring, and biopsies taken during endoscopy. HRM and 24-hour $\mathrm{pH}$ monitoring require a separate procedure and are associated with increased cost.

Imaged-enhanced endoscopy technology has progressed rapidly in the last decade. Since the development of fiber-optic endoscopy in 1963, four eras have defined gastrointestinal endoscopy. ${ }^{20}$ The last era of "high-definition imaging magnifying video endoscopy and improved equipment-based image-enhanced endoscopy", started in the 2010s and enabled endoscopist to detect and characterize lesions more precisely. Image-enhanced technology, like NBI, i-scan optical en- 
hancement, blue laser imaging, and linked color imaging, may improve visualization of mucosal changes during acid reflux. These diagnostic methods may differentiate NERD, ERD, and patients with reflux hypersensitivity or functional heartburn without HRM, 24-hour pH monitoring, or biopsy. One study, which used NBI, revealed that a higher proportion of patients with GERD had increased IPCLs and micro erosions. ${ }^{21}$ CLE was introduced with a novel endoscopic method that enables observation of the lesion in real-time and in vivo, suggesting the direction of target biopsy and treatment. ${ }^{22}$

The present study was based on an in vivo and ex vivo investigation of subtle histological changes in EGJ using pCLE. Our study compared the in vivo and ex vivo CLE findings of the ERD and NERD groups. The study was designed to identify in vivo and ex vivo findings, since screening ex vivo mucosectomy material using pCLE may provide a chance to accurately detect differences between patients with NERD and ERD. Although endoscopy procedure time was lengthened for the in vivo investigation, an ex vivo study poses no time limitations on the researcher, and does not have an adverse effect on the patient associated with the lengthened endoscopy procedure (decreasing oxygen saturation, bradycardia, etc.).

Refluxed acid and pepsin impair the protein of the junctional structure bound to the esophageal epithelium. Due to changes in membrane permeability, the acid is diffused into the epithelial cells, resulting in an inflammatory response. Thereafter, hyperplasia and papilla elongation of squamous basal cells occur. In addition, when the permeability is altered as a result of acid exposure, chloride $\left(\mathrm{Cl}^{-}\right)$, and water move into the cell, and DISs form due to the increase of bloodborne fluorescein in the intracellular space as seen on a pCLE image. ${ }^{14}$ Increased numbers and diameters of IPCLs and DISs can be considered a sensitivity marker for GERD-related tissue damage. It is also observed in NERD as well as in ERD (ERD has a higher detection rate than NERD), and some studies have shown that it is also observed in controls. ${ }^{15}$ In this study, DISs did not differ significantly between the ERD and NERD groups on the in vivo examination, but there was a significant difference between the ERD and control groups, and between the NERD and control groups. Also, the IPCL diameter was significantly larger in the ERD group than in the NERD group. Additionally, the number and CSA of the IPCLs were significantly higher in the ERD group than in the NERD group, and differed significantly between the two groups. In addition, studies by Dunbar et al. observed that when the PPI was administered, the DIS disappeared and then reappeared when the drug was discontinued. ${ }^{13,14}$ However, our study did not measure these changes, because we observed pCLE in patients who underwent ARMS after PPI discontinuation. Further studies are needed to determine the PPI effect by observing microscopic changes (such as DIS) using pCLE during NERD treatment. ${ }^{10}$ Repeated long-term acid reflux may lead to adverse events, such as Barrett's esophagus, dysplasia, and cancer, so real-time monitoring through pCLE will be helpful for detection of lesions without the need for biopsy. ${ }^{23,24}$ No dysplasia or cancerous lesions were noted in this study.

Our study does have limitations that may restrict the interpretation of the study. First, the pCLE technology allows for observation of only a narrow area. To overcome this problem, the endoscopy procedure time was lengthened. This way, the endoscopist searched the wide area of the distal esophagus with the narrow scope of pCLE. Therefore, the procedure time was approximately three times longer than that of a standard endoscopy procedure. It seems that this technology may not be suitable for daily practice. Artificial intelligence (AI) has gained popularity in clinical medicine. Applications of AI may be useful to detect early neoplastic or dysplastic lesions in gastrointestinal endoscopy. ${ }^{25}$ Computer-based algorithms may be added to the existing technology of pCLE. Detecting dilatation of DIS in the epithelium, counting the number of IPCLs, measuring the diameter and CSA of IPCLs, and detecting the irregularly shaped IPCL may be coded as the tasks of the software of pCLE. AI may shorten the time for diagnosing ERD or NERD via pCLE. Many clinical pilot studies must be carried out before development of this technology.

Another limitation of this study is the small sample size for each group. The reason for the small sample size may be due to a reduced number of patients requiring ARMS for the treatment of refractory GERD despite PPI. Another explanation could be the cost of the CLE probe and the procedure of ARMS, since these are not yet covered by the insurance companies in South Korea, and some patients could not afford the associated cost. Furthermore, ARMS has emerged as a new method, and it is not accepted as a standard treatment method for refractory GERD. Therefore, some patients did not give informed content due to the possible adverse effects associated with the ARMS procedure.

In conclusion, CLE may help detect microscopic changes in the esophagus beyond conventional endoscopy. There are currently no reference values for DISs and IPCLs. The present study includes a small patient population; thus, a large longitudinal study is needed to establish CLE as a diagnostic tool. Also, the measurement process was subjective. Thus, it will be necessary to measure, compare, and analyze the data of several people to reduce any statistical errors. We believe that objective criteria should be presented to be used as a reference. 


\section{Conflicts of Interest}

Joo Young Cho has been a Senior Consultant of Clinical Endoscopy; however, he was not involved in the peer reviewer selection, evaluation, or decision process of this article. The other authors have no potential conflicts of interest.

\section{Funding}

None.

\section{Author Contributions}

Conceptualization: Dong Keon Yon

Data curation: Eunju Jeong, In Kyung Yoo, DKY

Formal analysis: DKY

Investigation: EJ, IKY

Methodology: EJ, IKY

Supervision: Joo Young Cho

Writing-original draft: IKY, Abdullah Özgür Yeniova

Writing-review\&editing: JYC

\section{ORCID}

Eunju Jeong:

In Kyung Yoo:

Abdullah Özgür Yeniova:

Dong Keon Yon:

Joo Young Cho: https://orcid.org/0000-0002-7413-0604 https://orcid.org/0000-0003-0909-339X https://orcid.org/0000-0003-1681-364X https://orcid.org/0000-0003-1628-9948 https://orcid.org/0000-0001-9085-0313

\section{Supplementary Materials}

Video 1. In vivo confocal laser endomicroscopy procedure (https://doi org/10.5946/ce.2020.040v001).

Video 2. Ex vivo confocal laser endomicroscopy procedure (https://doi. org/10.5946/ce.2020.040v002).

\section{REFERENCES}

1. Kim JH, Park H, Lee YC. Is minimal change esophagitis really part of the spectrum of endoscopic findings of gastroesophageal reflux disease? A prospective, multicenter study. Endoscopy 2011;43:190-195.

2. van Malenstein H, Farré R, Sifrim D. Esophageal dilated intercellular spaces (DIS) and nonerosive reflux disease. Am J Gastroenterol 2008;103:1021-1028.

3. Armstrong D, Bennett JR, Blum AL, et al. The endoscopic assessment of esophagitis: a progress report on observer agreement. Gastroenterology 1996;111:85-92.

4. Kahrilas PJ. Diagnosis of symptomatic gastroesophageal reflux disease. Am J Gastroenterol 2003;98(3 Suppl):S15-S23.

5. Miwa H, Yokoyama T, Hori K, et al. Interobserver agreement in endoscopic evaluation of reflux esophagitis using a modified Los Angeles classification incorporating grades $\mathrm{N}$ and $\mathrm{M}$ : a validation study in a cohort of Japanese endoscopists. Dis Esophagus 2008;21:355-363.

6. Edebo A, Tam W, Bruno M, et al. Magnification endoscopy for diagnosis of nonerosive reflux disease: a proposal of diagnostic criteria and critical analysis of observer variability. Endoscopy 2007;39:195-201.

7. Wasielica-Berger J, Kemona A, Kiśluk J, et al. The added value of mag- nifying endoscopy in diagnosing patients with certain gastroesophageal reflux disease. Adv Med Sci 2018;63:359-366.

8. Kiesslich R, Gossner L, Goetz M, et al. In vivo histology of Barrett's esophagus and associated neoplasia by confocal laser endomicroscopy. Clin Gastroenterol Hepatol 2006;4:979-987.

9. Polglase AL, McLaren WJ, Skinner SA, Kiesslich R, Neurath MF, Delaney PM. A fluorescence confocal endomicroscope for in vivo microscopy of the upper- and the lower-GI tract. Gastrointest Endosc 2005;62:686-695

10. Gheorghe C, Iacob R, Becheanu G, Dumbrav Abreve M. Confocal endomicroscopy for in vivo microscopic analysis of upper gastrointestinal tract premalignant and malignant lesions. J Gastrointestin Liver Dis 2008; 17:95-100.

11. Ismail-Beigi F, Horton PF, Pope CE 2nd. Histological consequences of gastroesophageal reflux in man. Gastroenterology 1970;58:163-174.

12. Tobey NA, Carson JL, Alkiek RA, Orlando RC. Dilated intercellular spaces: a morphological feature of acid reflux--damaged human esophageal epithelium. Gastroenterology 1996;111:1200-1205.

13. Xue Y, Zhou LY, Lin SR. Dilated intercellular spaces in gastroesophageal reflux disease patients and the changes of intercellular spaces after omeprazole treatment. Chin Med J (Engl) 2008;121:1297-1301.

14. Dunbar KB, Agoston AT, Odze RD, et al. Association of acute gastroesophageal reflux disease with esophageal histologic changes. JAMA 2016;315:2104-2112.

15. Kim SE, Kim N, Oh S, et al. Predictive factors of response to proton pump inhibitors in Korean patients with gastroesophageal reflux disease. J Neurogastroenterol Motil 2015;21:69-77.

16. Inoue $\mathrm{H}$, Ito $\mathrm{H}$, Ikeda $\mathrm{H}$, et al. Anti-reflux mucosectomy for gastroesophageal reflux disease in the absence of hiatus hernia: a pilot study. Ann Gastroenterol 2014;27:346-351.

17. Sifrim D, Zerbib F. Diagnosis and management of patients with reflux symptoms refractory to proton pump inhibitors. Gut 2012;61:13401354.

18. Frazzoni M, Piccoli M, Conigliaro R, Frazzoni L, Melotti G. Laparoscopic fundoplication for gastroesophageal reflux disease. World J Gastroenterol 2014;20:14272-14279.

19. Yoo IK, Ko WJ, Kim HS, et al. Anti-reflux mucosectomy using a cap-assisted endoscopic mucosal resection method for refractory gastroesophageal disease: a prospective feasibility study. Surg Endosc 2020;34:11241131.

20. Ho SH, Uedo N, Aso A, et al. Development of image-enhanced endoscopy of the gastrointestinal tract: a review of history and current evidences. J Clin Gastroenterol 2018;52:295-306.

21. Lv J, Liu D, Ma SY, Zhang J. Investigation of relationships among gastroesophageal reflux disease subtypes using narrow band imaging magnifying endoscopy. World J Gastroenterol 2013;19:8391-8397.

22. Goetz M. Endomicroscopy and targeted imaging of gastric neoplasia. Gastrointest Endosc Clin N Am 2013;23:597-606.

23. Bertani H, Frazzoni M, Dabizzi E, et al. Improved detection of incident dysplasia by probe-based confocal laser endomicroscopy in a Barrett's esophagus surveillance program. Dig Dis Sci 2013;58:188-193.

24. Canto MI, Anandasabapathy S, Brugge W, et al. In vivo endomicroscopy improves detection of Barrett's esophagus-related neoplasia: a multicenter international randomized controlled trial (with video). Gastrointest Endosc 2014;79:211-221.

25. Urban G, Tripathi P, Alkayali T, et al. Deep learning localizes and identifies polyps in real time with $96 \%$ accuracy in screening colonoscopy. Gastroenterology 2018;155:1069-1078.e8. 